\title{
Search for Leptoquarks and 4th generation Quarks at CDF
}

\section{Aron Soha ${ }^{* \dagger}$}

University of California at Davis, USA

E-mail: soha@fnal.gov

At Run II of the Fermilab Tevatron, the CDF experiment provides good sensitivity for either discovery or setting limits on $1^{\text {st }}$ and $2^{\text {nd }}$ generation scalar, or $3^{\text {rd }}$ generation vector, leptoquark pair-production, where each leptoquark decays to a charged lepton and a quark with variable branching ratio $\beta$, or decays to a neutrino and quark with branching ratio $(1-\beta)$. By comparison with the theoretical expectations, we set mass limits, in some cases as a function of $\beta$. New quark generations are predicted in various scenarios for physics beyond the Standard Model. Here we include results from searches for $t^{\prime}$ and for $b^{\prime}$ production. The $t^{\prime}$ is searched for in decays to $W q$ and the $b^{\prime}$ in decays to $Z^{0} b$. All of these measurements are based on an integrated luminosity of $200-350 \mathrm{pb}^{-1}$, taken at $\sqrt{s}=1.96 \mathrm{TeV}$.

International Europhysics Conference on High Energy Physics

July 21st - 27th 2005

Lisboa, Portugal

\footnotetext{
* Speaker.

${ }^{\dagger}$ On behalf of the CDF collaboration.
} 


\section{Leptoquark Searches}

The striking parallels between the three generations of the quark and lepton sectors suggest that there may be connections at higher energies, possibly above the top quark mass. The experimental reach for proposed $1^{\text {st }}, 2^{\text {nd }}$, and $3^{\text {rd }}$ generation leptoquarks (LQ1, LQ2, and LQ3) has been studied (see [1, 2], and references therein) for both hadron and lepton machines. Here we report on the results from CDF at Run II of the Fermilab Tevatron.

A signature of two electrons and two jets is used to search for LQ1 in the eeqq channel. The selection seeks two isolated electrons with $E_{T}>25 \mathrm{GeV}$, where $E_{T}$ is the transverse energy, and vetoes on di-electron candidate invariant masses $\left(m_{e e}\right)$ in the range $76<m_{e e}<110 \mathrm{GeV} / \mathrm{c}^{2}$. There are also requirements on two isolated jets of $E_{T}>30 \mathrm{GeV}$ and $E_{T}>15 \mathrm{GeV}$. To combat Drell-Yan and top background, a two-dimensional cut is placed in the plane of $E_{T}\left(\right.$ jet $\left._{1}\right)+E_{T}\left(\right.$ jet $\left._{2}\right)$ versus $E_{T}\left(e_{1}\right)+E_{T}\left(e_{2}\right)$. The acceptance after this selection is $32-43 \%$ depending on the LQ1 mass. Backgrounds include $1.90 \pm 0.44$ events from $\mathrm{Z}+2 \mathrm{jets}, 0.35 \pm 0.03$ events from top dilepton, and $3.96 \pm 2.01$ events from fakes, with a total background contribution of $6.24 \pm 2.16$ events. The measurement is optimized for sensitivity for $\beta=\operatorname{Br}(\mathrm{LQ} \rightarrow \ell q)=1$, and in a data sample of $203 \mathrm{pb}^{-1}, 4$ events are seen, consistent with the backgrounds. The $95 \%$ confidence level (C. L.) limit is $m_{\mathrm{LQ} 1}>235 \mathrm{GeV} / c^{2}$.

To search for LQ1 in the evqq channel, with a signature of one isolated electron and two isolated jets, the following requirements are made: one electron with $E_{T}>25 \mathrm{GeV}$ and no second electron candidate; $E_{T}>60 \mathrm{GeV}$, where $E_{T}$ is the missing transverse energy of the event; 2 jets with $E_{T}>30 ; \Delta \phi\left(E_{T}\right.$, jet $\left._{i}\right)>10^{\circ}$, where $i=1,2 ; E_{T}\left(\right.$ jet $\left._{1}\right)+E_{T}\left(\right.$ jet $\left._{2}\right)>80 \mathrm{GeV}$; and $m_{T}(e, v)>120 \mathrm{GeV} / c^{2}$, where $m_{T}(e, v)$, the transverse mass combination of the electron candidate and missing energy, is used to suppress a background from $\mathrm{W}+2 \mathrm{jets}$. Two additional quantities, the $\mathrm{jet}+E_{T}$ transverse mass and the e+jet invariant mass, are used to place $3 \sigma$ window cuts around the expected LQ1 mass. The measurement is optimized for $\beta=0.5$ and the final acceptance is $2-25 \%$ depending on the assumed LQ1 mass. The resulting 95\% C. L. mass limit is $m_{\mathrm{LQ} 1}>176 \mathrm{GeV} / c^{2}$.

The signature and selection for the LQ1 search in the $v v q q$ channel consist of missing transverse energy $\left(E_{T}>60 \mathrm{GeV}\right)$ and two isolated jets (with $E_{T}>40 \mathrm{GeV}$ and $E_{T}>25 \mathrm{GeV}$ ). In addition, there must be no electron or muon candidates, and the angle between the two jets must satisfy $80^{\circ}<\Delta \phi\left(\right.$ jet $_{1}$, jet $\left._{2}\right)<165^{\circ}$. This measurement is able to exclude, with $95 \% \mathrm{C}$. L., LQ1 in the mass range $78-117 \mathrm{GeV} / c^{2}$.

A Bayesian approach is used to extract a combined LQ1 mass limit that takes input from the three channels discussed above, thus providing a mass limit over a range of $\beta$. To form the limit, the product likelihood is constructed from the individual channel likelihoods, 10,000 pseudoexperiments are run at each mass point, and the signal and background yields are smeared by their uncertainties. Furthermore, the correlations among channel selections are taken into account for the acceptance uncertainties. The resulting LQ1 mass limit as a function of $\beta$ is shown in figure 1 .

For our search for LQ2, the signature and selection for the $\mu \mu q q(\mu v q q)$ channel is similar to that of the eeqq (evqq) channel and omitted here. With the measurement optimized for $\beta=1$ ( $\beta=0.5$ ), the $95 \%$ C. L. mass limit is $m_{\mathrm{LQ} 2}>224(170) \mathrm{GeV} / c^{2}$. The combined LQ2 mass limit uses the same procedure as that given above for LQ1, and includes the $v v q q$ channel, in addition to $\mu \mu q q$ and $\mu v q q$. The resulting LQ2 mass limit as a function of $\beta$ is shown in figure 1 . 

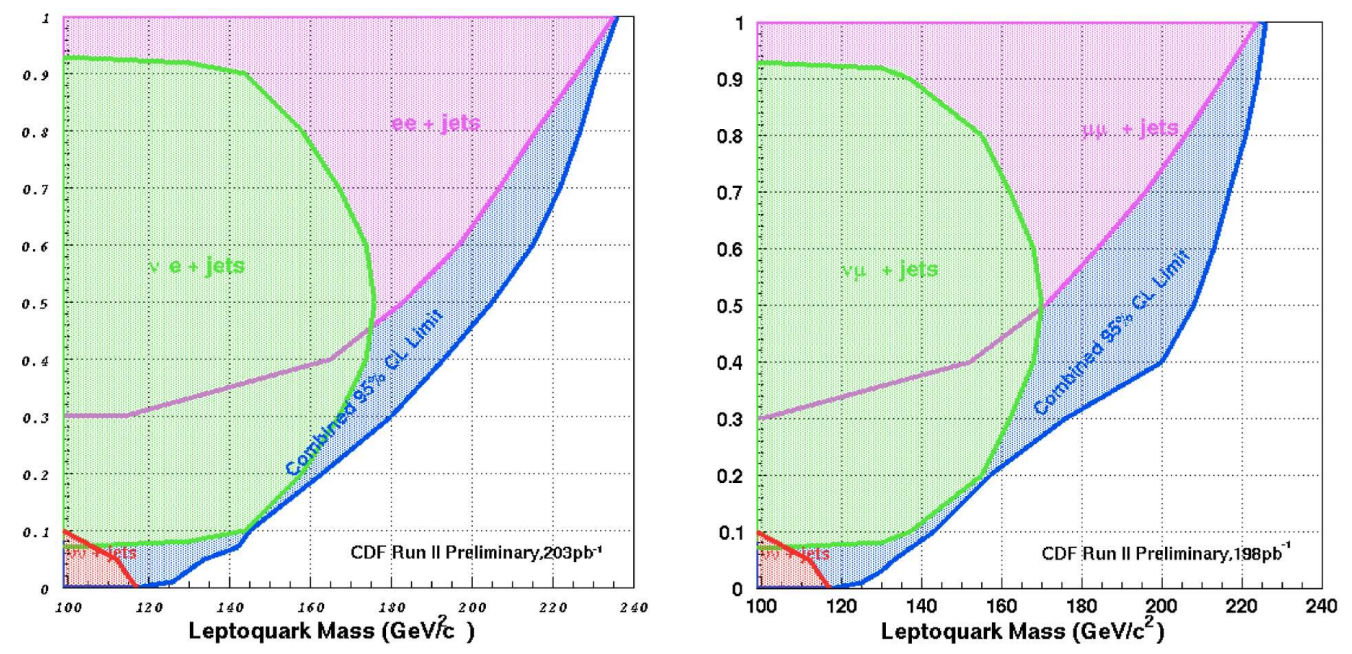

Figure 1: Combined mass limits as functions of $\beta$ for LQ1 (left) and LQ2 (right).

We are searching for $3^{\text {rd }}$ generation vector leptoquarks (VLQ3) in the channel $\tau \tau q q$. Various species of VLQ3 may appear, and we look for those that decay to $\tau \bar{b}$ (charge $-\frac{2}{3}$ ). We consider the final state where one of the taus decays to an electron or muon and the other tau decays hadronically $\left(\tau_{\mathrm{h}}\right)$. This represents $46 \%$ of the possible tau decay combinations. The signature and selection consist of one isolated electron (muon) with $E_{T}>10 \mathrm{GeV}\left(p_{T}>10 \mathrm{GeV} / c\right)$, one $\tau_{\mathrm{h}}$ (seeded by an isolated track) with $p_{T}>15 \mathrm{GeV} / c$, and two jets with $E_{T}>15 \mathrm{GeV}$. A new Monte Carlo simulation tool has been developed to simulate the production and decay of vector leptoquarks, properly taking into account the helicity amplitudes, and propagating the angular information to the produced taus. At the time of the conference, this analysis is in progress. Given the simulated cross section, the expectation is to be sensitive to seeing about three VLQ3 events with a mass around $300-$ $320 \mathrm{GeV} / \mathrm{c}^{2}$ if it exists in a sample of $319 \mathrm{pb}^{-1}$. This will surpass the existing limits, including that of $m_{\mathrm{VLQ} 3}>225 \mathrm{GeV} / c^{2}$ from $110 \mathrm{pb}^{-1}$ of CDF Run I data [3].

\section{Search for $4^{\text {th }}$ Generation $b^{\prime}$ Quark}

Our search for a $b^{\prime} 4^{\text {th }}$ generation quark is part of a more general search for a long lived particle that decays to $Z^{0}$. The strategy is to find a $Z^{0} \rightarrow \mu^{+} \mu^{-}$vertex and study the decay length, while keeping the search inclusive by looking for just one displaced $Z^{0}$ (by contrast, pair-production of $b^{\prime}$ quarks would lead to two $Z^{0} \mathrm{~s}$ ). The relevant selection consists of two muons with $p_{T}>20 \mathrm{GeV} / c$ and a di-muon candidate invariant mass of $81<m_{\mu \mu}<101 \mathrm{GeV} / c^{2}$. In addition, there is a requirement $\Delta \phi<175^{\circ}$ for the angle between the muon track candidates. This is because in back-to-back events, a small mis-measurement of the impact parameter can lead to a large mis-measurement of $L_{x y}$, which is the distance between the $Z^{0}$ decay vertex and the beam in the transverse plane. It is the $L_{x y}$ distribution that is used to define a control region and to search for an excess of signal over background. The main backgrounds are Standard Model $Z^{0}$ production, where one of the muon tracks is mis-reconstructed, and semileptonic $B$ meson decays. The number of events seen in $163 \mathrm{pb}^{-1}$ is consistent with background, and the excluded region is shown in figure 2, with and without an additional cut of $p_{T}>30 \mathrm{GeV} / c$ on the $Z^{0}$ candidates, since models favor high $p_{T}$. 

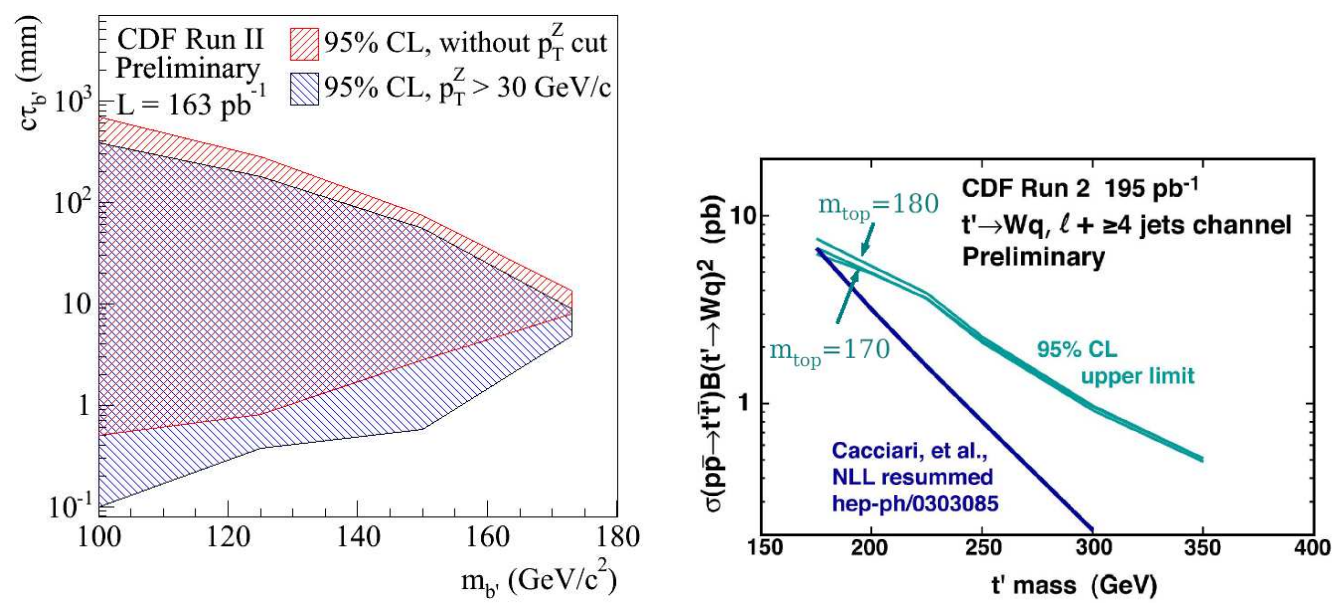

Figure 2: Results of 4th generation quark searches. Excluded region in decay length versus $b^{\prime}$ mass (left) and cross section times branching ratio as a function of $t^{\prime}$ mass (right).

\section{Search for $4^{\text {th }}$ Generation $t^{\prime}$ Quark}

A $4^{\text {th }}$ generation $t^{\prime}$ quark would be consistent with electroweak data and a heavy Higgs model [4]. It may seem natural to assume $m_{t^{\prime}}>m_{W}+m_{b^{\prime}}$ and search for $t^{\prime} \rightarrow W b^{\prime}$, however precision electroweak measurements suggest that $m_{t^{\prime}}$ and $m_{b^{\prime}}$ may be similar, so we search for $t^{\prime} \rightarrow W b, t^{\prime} \rightarrow W s$, and $t^{\prime} \rightarrow W d$, which are singly, doubly, and triply Cabibbo suppressed, respectively. The signature is $p \bar{p} \rightarrow t^{\prime} \bar{t}^{\prime} \rightarrow W q W \bar{q}$, where one of the $\mathrm{W}$ bosons decays through $W \rightarrow q \bar{q}$ and the other decays through $W \rightarrow \ell v$. Therefore, the selection is for an electron or muon of $p_{T}>20 \mathrm{GeV} / c$, event $E_{T}>20 \mathrm{GeV}$, and four jets with $E_{T}>15 \mathrm{GeV}$. No $b$-tagging is required, to keep the search sensitive to $t^{\prime} \rightarrow W s$ and $t^{\prime} \rightarrow W d$. We use the kinematic quantity $H_{T}$, which is the scalar sum $E_{T}^{\text {lepton }}+E_{T}+E_{T}^{\text {jets }}$, to separate signal from background. The fit to data includes components for background (including $t \bar{t})$ and the signal $\left(t^{\prime} \bar{t}^{\prime}\right)$. Results for $\sigma\left(p \bar{p} \rightarrow t^{\prime} \bar{t}^{\prime}\right) \times \operatorname{Br}\left(t^{\prime} \rightarrow W q\right)^{2}$ versus $t^{\prime}$ mass are shown in figure 2 . With $195 \mathrm{pb}^{-1}$ of data used in this search we are unable to limit $t^{\prime}$ production, but a larger sample is now available.

\section{References}

[1] J. L. Hewett and S. Pakvasa, Leptoquark Production In Hadron Colliders, Phys. Rev. D 37 (1988) 3165.

[2] W. Buchmüller, R. Rückl and D. Wyler, Leptoquarks In Lepton Quark Collisions, Phys. Lett. B 191 (1987) 442.

[3] F. Abe et al. Search for third generation leptoquarks in anti-p p collisions at $s^{* *}(1 / 2)=1.8-\mathrm{TeV}$, Phys. Rev. Lett. 78 (1997) 2906.

[4] H. J. He, N. Polonsky and S. f. Su, Extra families, Higgs spectrum and oblique corrections, Phys. Rev. D 64 (2001) 053004 [hep-ph/ 0102144$].$ 